\title{
Is Negative Feedback Better than No Feedback? The Impact of Social Dynamics on Reviewers' Review Decisions
}

\author{
Wenqi Shen \\ Pamplin College of Business \\ Virginia Tech \\ shenw@vt.edu
}

\author{
Yan Liu \\ Mays Business School \\ Texas A\&M University \\ yliu@mays.tamu.edu
}

\begin{abstract}
Consumers are increasingly relying on online product reviews when making purchase decisions. While the impact of online reviews on product sales has been studied extensively, only a few studies examine online reviewer's decision making. This paper directly measures social influences on reviewers' review decisions as well as on consumers'voting decisions (i.e. consumers vote for the helpfulness of reviews). Contrary to traditional cognitive evaluation theory, we find that both positive and negative feedback may positively motivate reviewers review behaviors. Receiving negative feedback may pose a challenge to reviewers and indicate the amount of attention the review receives, which could increase intrinsic motivation for reviewers to write new reviews. In addition, our results of the consumer voting decision model show that there is a multi-audience effect among consumers, as voters, who try to balance the sentiment of existing votes.
\end{abstract}

\section{Introduction}

Online product reviews have played a large role in consumer's decision-making process. Online reviews can facilitate the exchange of information and help consumers make informed decisions [34]. Therefore, potential buyers are increasingly relying on the information from online reviews to make purchase decisions. Many studies have shown that online reviews could significantly affect consumers buying behaviors and thus affect product sales [7, 13, 33].

Although we are aware of the significant impact of online reviews on product sales, not much research has been done to investigate the factors affecting reviewers' contributions over time. How do reviewers make review decisions? What factors may drive reviewers' voluntary review behaviors? Prior studies suggest that when lacking direct monetary incentives, social incentives such as peer recognition and attention are perceived as important social capital to online community members [28, 30]. Online community members are mainly motivated by social incentives when there is a lack of direct monetary rewards [30, 37, 39]. Similarly, online reviewers are likely to be motivated by social incentives since reviewers usually do not receive any direct monetary rewards for providing reviews. However, we are not well aware of how reviewers are influenced by such social incentives when making review decisions over time.

Only a few studies in the existing literature examine social dynamics or social incentives in online reviews. [40] showed that users' product ratings are influenced by social dynamics such as ratings posted by others previously. [34] found that previous rating variance could affect future ratings. They attribute the finding to a multiple-audience effect. When consumers face a highly varied audience, they are more likely to offer moderate opinions to avoid alienating any one segment of the audience [18]. However, the focus of these studies is still among ratings or reviewer-reviewer social influences, and the impact of ratings on sales. Many online review systems such as Amazon, Barnes and Noble, Yelp, or TripAdvisor offers a voting mechanism that allows potential consumers to provide feedback on the helpfulness of reviews. Such mechanism enables social interaction between reviewers who provide review content and consumers as voters who assess the quality of the reviews and make a vote. Different from the prior studies, we focus on Consumer-Reviewer dynamic social interactions and directly examine reviewer's decision-making process by investigating how different social factors, including review feedback and reviewer's online reputation, affect reviewer's review decisions.

In addition, we are interested in measuring the impact of social dynamics among review feedback since review feedback could potentially be one of the key factors that affect reviewer's review decisions. Prior studies show that different review characteristics including review text such as readability of the text [22, $29,35,36]$, review ratings such as the extremeness of 
the ratings [19, 29, 35] and reviewers such as reviewer identity or reputation $[19,22,29]$ may affect the perceived helpfulness of a review and thus affect the helpfulness votes that a review may receive. However, the major measurements in these studies are static and time invariant and they have not considered the impact of social dynamics among votes, which is the focus of this study. If voters are solely influenced by these known factors, they should be impacted in the same way regardless of when they vote for the helpfulness of the reviews since these factors are time invariant. Rather, the helpfulness of the reviews evolves over time. A previously voted helpful review may not always receive helpful votes from future voters. This indicates that there should be a dynamic factor that affects voter's decisions in addition to those static factors as studied in the literature. We draw upon findings from Moe and Trusov [34] that reviewers' ratings can be influenced by social dynamics and argue that consumers' perceived helpfulness of the reviews could be influenced by such social dynamics. Studies in online community suggest that under social pressure community users may follow the community consensus and vote for the same helpfulness for the review [28, 30]. However, both [34] and [40] find a multi-audience effect among online reviews that reviewers tend to balance among the existing opinions. With the mixed findings in the literature, it is interesting to examine how social dynamics influence consumers' perceived helpfulness of reviews.

In this study, we try to examine (1) the impact of social influence on consumers' perceived helpfulness of reviews and (2) the impact of social feedback, as the helpfulness votes of reviews, and online reputation, as the rank of reviewers, on reviewers' review decisions. This study draws upon theories in information systems and marketing literatures that social dynamics play an important role in online users' decision-making process. Community members' decisions are likely to be affected by social interactions such as peer recognition and online reputation. Similarly, in the online review context, reviewers' decisions to post a review could be influenced by the social dynamics of a review system as well.

We collect two data sets from Amazon.com to study the two research questions. One is on review level and the other is on reviewer level. Our unique panel datasets include daily helpfulness votes on each review and daily review activities for each reviewer. The two datasets cover a three-month period which allows us to observe how reviewers make review decisions over time. We find that social dynamics do have a strong influence on consumers' helpfulness votes as well as reviewer's review decisions. At review level, consistent with the findings in literature, our results indicate a multi- audience effect among helpfulness votes. The arrival of future helpfulness vote is affected by the number of existing votes in the opposite sentiment. The more negative or unhelpful votes the review currently has, the more likely consumers as the voters will vote it positively. Similarly, the more positive or helpful votes the review has, the more likely future voters will vote it negatively. Our results show that existing votes do have an impact on the direction of future votes, and more interestingly, voters tend to balance among existing votes through adding a vote in the opposite sentiment.

On the reviewer level, we find that both social feedback and online reputation significantly affect reviewers' review decisions. The results show that receiving votes is one of the key factors drive reviewer's review decisions. Interestingly, we find that both positive and negative feedback increases the probability for reviewers to write a new review, which is contrary to Deci's cognitive evaluation theory that positive feedback increases individual's intrinsic motivation and negative feedback decreases intrinsic motivation [11, 12]. However, this discrepancy can be theoretically explained in two ways. First, when reviewers are intrinsically interested in writing reviews, such negative feedback may not be perceived as unduly negative but as providing a challenge, which could increase reviewers' motivation of writing future reviews [41]. This is align with the general behavior theory that frustration following negative feedback is experienced only if expected reward is not forthcoming. When the task is intrinsically interesting to individuals, it provides a form of self-reinforcement and the challenge can be viewed as a form of persistence [1] which could account for the increase in motivation following negative feedback [41]. Second, in the information age, individual's attention becomes a new form of wealth as defined in the attention economy [10]. Consumers' attention can be viewed as an important social benefit to reviewers $[10,42]$. Receiving negative feedback at least indicates that people are reading the reviews and assessing them. As a result, the quantity of negative votes could still positively motivate reviewers in writing new reviews. This effect is more obvious when combining with the findings on receiving no votes. Our results show that receiving no votes strongly diminishes reviewer's intention to write new reviews. Receiving no votes at all discourages reviewers since it indicates zero social interaction and low readership of their reviews. Whereas receiving negative feedback could at least indicate readership of the reviews and can be perceived as posing a challenge or receiving attention, which increase reviewers' motivation to contribute. In addition to the effect on social feedback, we find that decrease in reviewer reputation increases the probability for reviewers to write new reviews. This suggest that 
reviewers recognize the value of their online reputation and work actively to maintain their reputation status through making review contributions.

The rest of the paper is organized as follows. In the next section, we review the previous studies in this field and discuss the differences between this study and the existing literature. Next, we introduce the empirical methodology used to address the research questions. Then, we present the results in section four. Finally, we conclude with a discussion of the implications and future research directions.

\section{Literature Review}

There is a growing interest in academia to investigate online user-generated content such as online reviews [7, 19, 31, 33, 44, 45], blogs [14, 15], or mobile content [21]. The majority of the prior studies focus on the impact of web content.

In the online review literature, researchers mainly examine the impact of online reviews on the consequent product sales [7, 33, 43]. They usually use three numerical measurements to assess the impact of online reviews: the volume, as the number of reviews, the variance, as the variance across ratings and the valence, as the average rating. A high volume of online reviews increases the awareness of a product. Thus, it can help to increase future sales [33]. The valence of online reviews is typically measured as the average ratings of online reviews. It conveys the attitudes of previous purchasers on average, which may or may not be sufficient to convince future buyers to purchase the product $[17,33,44]$. The findings are mixed on the role of variance in online review context. [46] finds that the variance of movie reviews does not play a significant role in box office revenues. However, [43] reports a significant role of review variance in sales especially when interacting with the valence, the average rating.

Recently, studies began to examine the impact of the source of online reviews, such as reviewers' identities or geographic locations, on potential buyers' decisions [19]. For example, [19] showed that reviews which disclose reviewers' identity information are perceived to be more helpful than reviews provided by anonymous reviewers. Thus, these reviews with reviewers' identity information would have stronger impact on product sales. These findings confirm the theory in social psychology literature that the source characteristics of the information can affect individuals' judgment $[2,4]$.

In addition, researchers have identified other important characteristics of online reviews, such as the quality of online reviews, which could potentially affect consumers' purchase decisions and product sales. The quality of the reviews is often measured as the ratio of the helpful votes to total votes for each review. This ratio indicates the usefulness and the credibility of the information as perceived by the readers [5]. A high ratio of helpfulness indicates higher quality of the review which is perceived as more useful information. Reviews with a high ratio of helpfulness are more persuasive than other reviews to entice potential buyers to try the product. Therefore, reviews with high ratio of helpfulness are positively associated with the subsequent product sales [5]. [5] used online helpfulness votes as an indicator of the review quality and found that consumers do pay attention to the quality of the reviews in addition to just the volume or the valence. [20] uses the helpfulness votes as an indicator of reviewers' review quality and finds that reviewers' review quality is relatively consistent over time. [32] studies the impact of information attributes in review context on consumers' purchasing decisions and suggests a negative effect from the great amount of conflicting information in reviews on sales.

Different from the above studies which focus on the impact of online reviews, this study investigates the antecedent of reviews, i.e. online reviewers' behaviors. It tries to understand the factors that affect reviewers' decisions on writing reviews. More importantly, it considers social dynamics on reviewers' review decisions. There is a small literature that directly studies social influences on reviews. [40] shows social influences in the context of online ratings through an experimental setting. It finds that reviewers who have decided to post their opinions tend to negatively adjust their product evaluations after reading negative reviews. Their findings indicate that consumer's review behavior could be affected by social context. [34] also finds that previously posted reviews can influence the ratings of future reviews which they attribute as social influence and social dynamics of rating behaviors.

In this study, we investigate beyond the social influence from previous ratings by considering social feedback and online reputation as two other important forms of social influences in affecting reviewers' decisions. Various prior researches pointed out that online users desire peer feedback or peer recognition which encourages them to keep contributing to online communities voluntarily $[28,30]$. More particularly, Deci's cognitive evaluation theory suggests that feedback may affect individual's motivation in their participations and may either increase or decrease an individual's intrinsic motivation on completing an assigned task $[11,12]$. Positive feedback may increase such intrinsic motivation and thus leads to high participation, whereas negative feedback may decrease one's intrinsic motivation and reduce the likelihood of participating. While such conclusions are based on experiments in an offline environment, we are not fully 
aware of the effect of feedback on individual's motivations in an online setting. In an online environment, individuals do not receive immediate or direct feedback from peers rather they receive feedback from other anonymous users through a virtual platform. We are not well aware of how social feedback motivate online users such as online reviewers to make contribution decisions. In this study, we empirically measure the role of social feedback and online reputation on reviewers' decision-making process. This study can help researchers to better understand how online reviewers interact with social influence in addition to the abovementioned findings in the existing literature. Our study can help solve the puzzle in online review literature on identifying what factors may motivate reviewers' voluntary contributions and in what way.

\section{Empirical Methodology}

\subsection{Data}

The goal of this study is to understand first how social dynamics affect future helpfulness vote and then how social feedback and online reputation affect reviewers' review decisions. We collected two datasets from Amazon to examine the effects of social dynamics at different levels, namely review level and reviewer level. At review level, we need daily voting behaviors for each review. Whereas at reviewer level, we need reviewers' daily review activities as well as the social feedback they receive and the changes of their online reputation.

At the review level, our sample includes all books released on Amazon in September and October 2010, which contains 1,751 books. We tracked the publicly available information on those books at amazon.com each day for a three-month period. One of the key benefits to use newly released products is for us to observe the impact of social dynamics from the initial stage where no other existing effects may potentially affect voters' behaviors. At the end of the three-month data collection period, our panel dataset has 690 books that have more than two reviews and we have 10,195 reviews in the data set.

At reviewer level, the dataset contains reviewers' daily activities. We collected daily information for 3,000 randomly selected reviewers from Amazon.com for a three-month period. The dataset includes key information on daily reviewer rank, helpful votes, total votes, and number of reviews they posted each day. At the end of the data collection period, our panel dataset has 687 reviewers who have posted more than two reviews during the data collection period.

\subsection{Empirical Model}

3.2.1. Review Feedback Model. The data we use to study the impact of social dynamics among review feedback is an unbalanced panel dataset for a threemonth period. Specifically, we separately investigate the social influences on positive feedback and negative feedback, which are measured using helpful votes and unhelpful votes. Thus, the two dependent variables are DailyHelpfulVotes and DailyUnhelpfulVotes, which are the number of helpful votes and unhelpful votes one review receives each day. Since these variables are count variables which violates the assumption of homoscedasticity and normal distribution of the errors under traditional Ordinary Least Square (OLS) model, we adopt a Poisson model that accounts for the discrete and non-negative nature of the count data $[24,16]$. We assume the arrival of the votes follows a Poisson distribution. In addition, since our data exhibits overdispersion, we estimate a negative binomial distribution model that accommodates over-dispersed data of count variables and has been applied in marketing and information systems literatures [26, 42].

The process of a review receiving votes can be captured by a Poisson process. Therefore, the probability of a review $i$ obtains vote type $j$ at time $t$ with vote arrival rate $\lambda_{i t}$ can be presented as:

$P_{j i t}\left(\boldsymbol{Y}_{j i t}=y \mid \lambda_{j i t}\right)=\frac{e^{-\lambda_{j i t}} \lambda_{j i t}^{y}}{y !}, y=0,1,2, \ldots$

where $j=1$ for helpful votes and 2 for unhelpful votes. $\boldsymbol{Y}_{\text {Iit }}$ is the DailyHelpfulVotes for review $i$ at time $t$, and $\boldsymbol{Y}_{2 i t}$ is the DailyUnhelpfulVotes. $y$ is the count of votes. To account for the social dynamics effects, we model the votes arrival rate following [16] and [24].

$$
\lambda_{j i t}=\exp \left(\boldsymbol{\gamma}_{i}+\boldsymbol{a}^{\prime} \boldsymbol{Z}_{i t}+\boldsymbol{\varepsilon}_{j i t}\right)
$$

where $\boldsymbol{Z}_{i t}$ is a vector of the explanatory variables and $\boldsymbol{a}$ is a vector of parameters. $\gamma_{i}$ is the review-specific fixed effects which controls for the intrinsic value of review $i$. $\varepsilon_{j i t}$ is the error term. We performed the Hausman [25] test to test the validation of using a fixed effects model or a random effects model. Our result rejects the null hypothesis at $1 \%$ significance level and is in favor of using a fixed effects model. Using fixed effects model allows the error term to be correlated with the explanatory variables and controls for time-invariant unobserved characteristics that are associated with each review which may affect the arrival rate of the votes.

To capture the social dynamics among review feedback, we construct three explanatory variables including HelpfulVotes it- $1_{\text {wh }}$ which is natural log of number of existing helpful votes for review $i$ at time $t$ - 


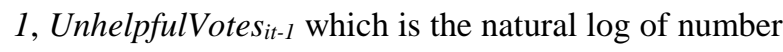
of existing unhelpful votes, and PerHelpfulVotes it-1 $_{1}$ which is the percentage of helpful votes over total votes for review $i$ at time $t$-1. HelpfulVotes and UnhelpfulVotes measure effect from the absolute quantity of existing votes on the arrival of future votes, whereas PerHelpfulVotes measures the effect from relative magnitude of the positive feedback on future feedback. The daily information in our dataset allows us to observe the existing votes that a voter sees before he/she votes, which reproduces the dynamic environment for us to examine the sequential influences on voters' decisions.

In addition, we control for review and reviewer characteristics that may potentially affect the perceived helpfulness of the review. We include Rating which is the star rating of the review. As previous study has found that negative reviews tend to have a stronger impact on consumer's purchase decisions and thus could be viewed as more helpful than positive ratings [7]. We also include a variable RatingDeviation, which is the squared difference between the average rating for the product at the time and the focal review's rating. As studies in online community have identified, deviated opinion may be discounted as it moves away from community consensus. Therefore, RatingDeviation controls for this potential discounting effect from a deviated rating. To control for reviewer characteristics,

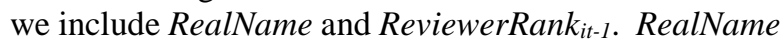
is a dummy variable which takes 1 if the reviewer reveals his/her true identity and 0 otherwise. ReviewerRank is the natural $\log$ of the reviewer rank at time $t$ - 1 . Note that Rating and RealName are both timeinvariant variables and all the other variables in the model changes each day.

3.2.2. Reviewer Decision Model. Next, we discuss the model that we use to measure social influences on reviewers. We develop a Proportional Hazard model (PHM) to assess the effects of social feedback and online reputation on reviewers' review decisions. PHM has been widely used in marketing literature to model consumer's inter-purchase time. Consumers are more likely to make purchases if their inventory is low. Thus, consumer's decisions on whether to buy are correlated with the time since their last purchase [8]. Similarly, in this study, we use the time since last review of a reviewer as the argument of the baseline hazard function in a PHM. In addition to the baseline hazard function, the PHM has a covariate function which captures the effects of social feedback and online reputation. Following [34], we use an exponential covariate function and derive the hazard function as follows:

$$
h\left(\tau_{j t} ; \lambda_{j}, \boldsymbol{\beta}_{j}\right)=\lambda_{j} * e^{X_{j t} \boldsymbol{\beta}_{j}}
$$

where $j$ is the reviewer index, $t$ is the time index, and $\boldsymbol{X}_{j t}$ is a vector of covariates. $\tau$ is the number of days elapsed since reviewer $j$ 's last review, which evolves as follows:

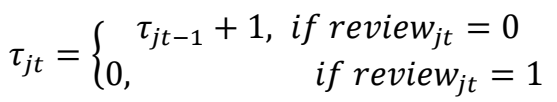

The hazard function describes the frequency of reviewer's review behavior. It can be decomposed into two parts, a baseline hazard rate $\lambda_{j}$ and a covariate function $e^{X_{j t} \beta_{j}}$. The baseline hazard rate indicates reviewers' review behaviors without the impact from the covariates. The coefficients in the covariate function capture the effect from the specific covariates we try to examine. Since we try to measure the effect of social feedback and online reputation, we construct the following main covariates:

DaysNoVotes $_{j t}$, which is the number of days that reviewer $j$ receives no votes by time $t$ since last vote, CumUnhelpfulVotes $s_{j}$, which is the number of unhelpful votes reviewer $j$ receives by time $t$ since last review,

CumHelpfulVotes s $_{t}$, which is the number of helpful votes reviewer $j$ receives by time $t$ since last review, CumPerHelpfulVotes ${ }_{j t}$, which is the percentage of helpful votes over total votes for reviewer $j$ by time $t$ since last review, and

DiffReviewerRank ${ }_{j t}$, which is defined as:

$$
\ln \left(\mid \text { ReviewerRank }_{j t}-\text { ReviewerRank }_{j t-1} \mid\right) * 1 \text {, if }
$$
ReviewerRank $_{j t}>$ ReviewerRank $_{j t-1}$

$\ln \left(\mid\right.$ ReviewerRank $_{j t}-$ ReviewerRank $\left._{j t-1} \mid\right) *-1$, if ReviewerRank $_{j t}<$ ReviewerRank $_{j t-1}$.

The first four covariates measure the effect from different type of social feedback. Since hazard model considers each event of writing new review(s) as a failure event, we calculate the cumulated votes since each time reviewer writes new review(s) to measure the exact effect from social feedback on each review decision. If reviewers were motivated by gaining online attention, receiving negative votes would still be better than receiving no votes. The coefficient for DaysNoVotes should be negative whereas the coefficient for CumUnhelpfulVotes should be positive. However, if gaining attention is not one of the primary motivations for reviewers to contribute, receiving negative feedback could decrease intrinsic motivations for reviewers to contribute. The coefficient of CumUnhelpfulVotes would be negative. The last covariate DiffReviewerRank measures the effect of changes in online reputation on reviewers' decisions. If reviewers are motivated by their online reputation, a change in reviewer rank can affect reviewers' review motivations and thus affect their review decisions. Note 
that all these covariates are time-varying which allows us to capture reviewers' dynamic review decisions.

In addition to the above five covariates, we also control for reviewer level characteristics such as reviewer's real name identity (RealName $)_{j}$ ) and reviewer's daily reviewer rank (ReviewerRank $k_{j t}$ ). These two variables are defined the same as in the review feedback model.

Table 1 shows a summary of the statistics of all the variables.

Table 1. Summary statistics

\begin{tabular}{lcccc}
\hline Variable & Mean & $\begin{array}{c}\text { Std. } \\
\text { Dev. }\end{array}$ & Min & Max \\
\hline Review Feedback Model & & & & \\
HelpfulVotes & .95 & .92 & 0 & 6.78 \\
UnhelpfulVotes & .55 & .79 & 0 & 5.55 \\
PerHelpfulVotes & .71 & .34 & 0 & 1 \\
Rating & 4.12 & 1.22 & 1 & 5 \\
RealName & .51 & .50 & 0 & 1 \\
ReviewerRank & 9.65 & 4.07 & 0 & 15.83 \\
RatingDeviation & .98 & 1.83 & 0 & 14.69 \\
\hline Reviewer Decision Model & & & \\
CumHelpfulVotes & 5.24 & 12.99 & 0 & 496 \\
CumUnhelpfulVotes & 1.57 & 4.12 & 0 & 100 \\
DaysNoVotes & 9.13 & 15.40 & 0 & 102 \\
CumPerHelpfulVotes & .72 & .16 & .06 & 1 \\
ReviewerRank & 10.16 & 2.81 & 2.48 & 15.73 \\
RealName & .41 & .49 & 0 & 1 \\
DiffReviewerRank & .92 & 3.27 & -15.64 & 15.66 \\
\hline
\end{tabular}

\section{Results}

\subsection{Review Feedback Model Results}

Our results show that social dynamics of votes can significantly affect the arrival of subsequence votes. Interestingly, we find that potential buyers, as the voters, tend to balance the existing opinions on the helpfulness of the reviews. As the results indicate, increases in the number of existing helpful votes tend to discourage future helpful votes, i.e. the coefficient for HelpfulVotes in Model (1) is negative. Similarly, increases in the number of existing unhelpful votes discourage future unhelpful votes, i.e. the coefficient for UnhelpfulVotes in Model (2) is negative. This finding is consistent with the multiple-audience effects that [34] has reported among review ratings. When facing a heterogeneous audience, reviewers tend to adjust the message to balance the existing opinion [40]. Our results suggest that similar effects may exist among review voters who may adjust their votes to balance the existing opinions on the helpfulness of the review. This effect is more obvious with the unhelpful votes as the percentage of existing helpful votes positively affects the number of future unhelpful votes, i.e. the coefficient of PerHelpfulVotes for Model (2) is positive. In other words, voters do not only pay attention to the amount of unhelpful votes but also account for the ratio of helpful votes when making an unhelpful vote. This provides a strong evidence suggesting that voters do balance between existing opinions before making the vote.

Table 2. Review Feedback Model Results

\begin{tabular}{|c|c|c|}
\hline Variables & $\begin{array}{c}\text { (1) } \\
\text { DailyHelpfu/Votes }\end{array}$ & $\begin{array}{c}\text { (2) } \\
\text { DailyUnhelpfuIVotes }\end{array}$ \\
\hline HelpfulVotes ${ }_{\text {it-1 }}$ & $\begin{array}{c}-1.727^{* *} \\
(.071)\end{array}$ & $\begin{array}{l}-.119 \\
(.126)\end{array}$ \\
\hline UnhelpfulVotes $_{\text {it-1 }}$ & $\begin{array}{l}.158^{*} \\
(.083)\end{array}$ & $\begin{array}{l}-1.202^{* *} \\
(.112)\end{array}$ \\
\hline PerHelpfuIVotes ${ }_{i t-1}$ & $\begin{array}{l}.301 \\
(.253)\end{array}$ & $\begin{array}{l}1.269^{* *} \\
(.314)\end{array}$ \\
\hline Rating $_{i t}$ & $\begin{array}{l}-.829^{* *} \\
(.257)\end{array}$ & $\begin{array}{l}-.205 \\
(.137)\end{array}$ \\
\hline RealName $_{i}$ & $\begin{array}{l}1.779^{* *} \\
(.432)\end{array}$ & $\begin{array}{l}.086 \\
(.361)\end{array}$ \\
\hline ReviewerRank ${ }_{i t-1}$ & $\begin{array}{c}-.014 \\
(.010)\end{array}$ & $\begin{array}{l}-.026^{* *} \\
(.011)\end{array}$ \\
\hline RatingDeviation $_{\text {it- } 1}$ & $\begin{array}{l}-.007 \\
(.055)\end{array}$ & $\begin{array}{l}.053 \\
(.054)\end{array}$ \\
\hline $\begin{array}{l}\text { Log Likelihood } \\
\text { Obs. }\end{array}$ & $\begin{array}{c}-5776.237 \\
31,742\end{array}$ & $\begin{array}{c}-3664.869 \\
22,505\end{array}$ \\
\hline
\end{tabular}

In addition, we find that the more negative the rating is, the more helpful votes the review will receive. This is consistent with previous studies that negative ratings have a stronger impact on consumer's buying decisions than positive ratings [7] and people tend to find negative reviews more helpful than positive reviews.

\subsection{Reviewer Decision Model Results}

Next, we discuss results from the reviewer model which examines the impact of social dynamics on reviewer's review decisions. As discussed above, we are particularly interested in two forms of social dynamics, the social feedback and reviewer's online reputation. Recall that we break down social feedback 
into positive feedback, negative feedback and no feedback, which are measured through number of helpful votes, number of unhelpful votes, and days with no votes. Interestingly, different from predictions based on cognitive evaluation theory that negative feedback may diminish people's intrinsic motivation on performing the task [12], we find that both positive and negative feedback positively affect the probability for reviewer to write reviews. The positive effect from negative feedback may be attributed to two possible explanations. First, when reviewers are intrinsically interested in the task of writing reviews, such negative feedback can be perceived as posing a challenge, which may increase their motivation of writing reviews [41]. In addition, receiving negative votes at least show that people are reading the reviews and assessing them, which helps reviewers to quantify the amount of attention their reviews obtain. Such attention can be viewed as a virtual capital reviewers may gain and becomes valuable social benefits that motivate reviewers to contribute to review sites [10, 27]. Our findings suggest that receiving social benefits may outweigh the negative effects from negative feedback on reviewer's motivations to continue writing reviews. In other words, reviewers care about the amount of attention their reviews may receive and are motivated through receiving votes from the readers.

In addition, the findings on the effect of receiving no votes further support the interpretation of the results on negative feedback. The negative coefficient of DaysNoVotes indicates that not receiving any votes definitely diminishes reviewer's motivation to write a future review. Receiving no votes suggest that a review may not have any readership which discourages reviewers that desire obtaining attention through writing reviews. These interesting yet counterintuitive findings coincide with the prediction under attention economy that attention is perceived as the most scarce but valuable resource in the information age [9, 23]. With a lack of direct monetary incentives, social incentives such as attention become a new form of wealth and can motivate reviewer's review contributions. Receiving social feedback as a mean to quantify the amount of attention seems to be one of the important factors that drive reviewer's review decisions online.
Table 3. Reviewer Model Results

\begin{tabular}{|c|c|c|c|}
\hline Variables & (1) & (2) & (3) \\
\hline CumHelpfulVotesjt-1 $_{\text {. }}$ & $\begin{array}{l}.008^{* *} \\
(.001)\end{array}$ & $\begin{array}{l}.008^{* *} \\
(.001)\end{array}$ & $\begin{array}{l}.008^{* *} \\
(.001)\end{array}$ \\
\hline CumUnhelpfulVotesjt-1 & $\begin{array}{l}.021^{* *} \\
(.004)\end{array}$ & $\begin{array}{l}.021^{* *} \\
(.004)\end{array}$ & $\begin{array}{l}.021^{* *} \\
(.003)\end{array}$ \\
\hline DaysNoVotes $_{j t-1}$ & $\begin{array}{l}-.016^{* *} \\
(.002)\end{array}$ & $\begin{array}{l}-.016^{* *} \\
(.002)\end{array}$ & $\begin{array}{l}-.019^{* *} \\
(.002)\end{array}$ \\
\hline CumPerHelpfulVotes $_{j t-1}$ & $\begin{array}{l}-.480^{*} \\
(.219)\end{array}$ & $\begin{array}{l}-.480^{*} \\
(.219)\end{array}$ & \\
\hline ReviewerRank $k_{t-1}$ & $\begin{array}{l}-.049^{* *} \\
(.013)\end{array}$ & $\begin{array}{l}-.042^{\star *} \\
(.013)\end{array}$ & \\
\hline RealName $_{j}$ & $\begin{array}{l}-.015 \\
(.043)\end{array}$ & $\begin{array}{l}-.019 \\
(.043)\end{array}$ & \\
\hline DiffReviewerRank $_{j t-1}$ & $\begin{array}{l}.023^{* *} \\
(.008)\end{array}$ & & \\
\hline $\begin{array}{l}\text { Log Likelihood } \\
\text { Obs. }\end{array}$ & $\begin{array}{c}-19704.697 \\
51,145 \\
\end{array}$ & $\begin{array}{c}-19709.398 \\
51,145 \\
\end{array}$ & $\begin{array}{c}-19723.206 \\
51,145 \\
\end{array}$ \\
\hline
\end{tabular}

In addition, changes in reviewer's online reputation play an important role in reviewer's review decisions. Our results show that reviewers become more likely to contribute when their reviewer rank decreases. Note that the higher the reviewer rank, the smaller the value of the rank is. Therefore, a positive value of DiffReviewerRank indicates a decrease in reviewer rank which positively affects reviewer's probability of writing a new review (i.e. the coefficient of DiffReviewerRank is positive). This suggests that reviewers treasure their online reputation and try to maintain their reputation status through writing reviews.

\section{Discussions and Conclusion}

This study is the first attempt to understand how social influences affect reviewers' review decisions and consumers' voting decisions. We utilize two unique datasets that contain dynamic daily information on reviews and reviewers' behaviors to measure the social impact on reviewer and consumer behaviors. Since online reviews play an important role in driving product sales and helpfulness votes may not only signal the quality of reviews but also motivate reviewers' review decisions, our results yield important theoretical and managerial contributions.

Theoretically, this is the first study as to our knowledge to investigate the effect of social dynamics among both review voters and reviewers and to directly measure the impact of social influences on consumers' voting decisions and on reviewers' review decisions. Different from the existing literature on online reviews, we examine the dynamic impact from existing opinions on future helpfulness of reviews, and from two social 
factors on reviewers' review decisions. We provide empirical evidence to address the mixed findings in the literature on how individual's assessment may be affected by existing opinions. We show that rather than following the community consensus, potential consumers seem to offer a balanced assessment on the helpfulness of reviews through voting for the opposite direction. Such effect is consistent with the multiaudience effect among reviewers' rating behaviors [34]. Previous studies often use the number of helpful votes as a measure to determine the quality or the value of a review [6, 29]. However, since voters may intent to balance the existing opinions by voting for the opposite sentiment, using such votes at a given time may not reveal the actual quality or the helpfulness of a review. Particularly, such helpfulness votes may evolve overtime and the aggregate sentiment of the votes could change accordingly. This may introduce a sample selection bias in the empirical results that are based on data collected at a randomly selected time point.

Interestingly, contrary to the cognitive evaluation theory, we find that reviewers' behaviors are in line with the general behavior theory that they may consider negative feedback as a challenge or a form of attention, which could increase their intrinsic motivation to review.

Furthermore, this study helps researchers to better understand what social factors may affect online users' contribution decisions. Our results provide empirical evidence on the importance of social feedback and online reputation in encouraging users' voluntary contributions. It extends the emerging research interests on understanding the motivations of users' voluntary contributions and how such motivations affect users' decisions. Furthermore, it addresses the mixed finding in the literature on how negative feedback may affect individuals' decisions and how existing opinions may affect future opinions among online consumers.

Practically, our results can help companies (e.g. Amazon) to better understand the factors that may affect reviewer's review decisions and to better design the review system to accommodate those social factors. We find that social dynamics do have a significant impact on reviewers' review decisions. In particular, the helpfulness votes may not only be used by potential consumers to assess the quality of reviews and identify helpful reviews, they could potentially offer a mean for reviewers to quantify the amount of attention their reviews receive. Reviewers may use such a tool as a form of social feedback from readers, which could positively motivate their review behaviors. Thus, it is wise for the companies to have a web system that encourages consumer's vote. Moreover, companies should consider providing a mechanism that helps reviewers better visualize the amount of attention their reviews receive, which in turn could increase users' contributions to the review system.

Moreover, we find that negative feedback is not always a discouraging sign but may increase reviewers' motivations to review. Companies should consider offering a channel for consumers to vote in both directions and should not be afraid from providing a channel for consumers to vote negatively of the reviews. This is because reviewers may not always view such negative feedback negatively. Currently, many social sites only offer a mean to let users vote positively for the content. For example, TripAdvisor only allows consumers to vote a review as helpful. Yelp provides three options for consumers to vote, "useful", "funny", and "cool", but they are all on the positive side. Even Amazon has changed their display after our data collection period to show only the helpful votes for reviews. Such strategy may not be optimal since reviewers lose the amount of additional attention their content could have received from the negative side of voters.

In addition, we find that the higher the number of existing positive votes, the lower the chances for the content to receive future positive votes. Offering a single channel for voters could potentially discourage future voters from both sides. Voters with positive opinions may choose not to put in another same vote. However, voters with negative opinions may not have the channel to vote at all. That is, closing the negative feedback channel could (1) lose those votes from the other side of the feedback and provide a biased assessment of the true quality of the reviews, and (2) eliminate the opportunity for reviewers to quantify additional attention their reviews may receive from consumers who would have voted negatively to the reviews. Such voting mechanism may not only provide biased assessment on reviews, but also potentially discourage voters to keep voting which may discourage reviewers to contribute over time. Companies may consider open the channel to allow reviewers receive social feedback from both directions to not only provide a holistic view of the quality of reviews but also allow reviewers to gather all the attention their reviews may receive. Alternatively, companies may consider providing a mechanism to help reviewer visualize the amount of readership on their reviews. This may provide a direct measure of the amount of attention their reviews receive and could potentially increase reviewers' motivates and their activity levels.

This research sheds light on examining social influences among reviews and reviewers. It would be of great interests to investigate such influences in other emerging fields such as social media or crowdfunding sites. In addition, the current analysis does not consider heterogeneity among consumers and reviewers. We 
will extend the models to allow heterogeneous consumers as well as reviewers. However, we do not expect the current results to be much different. We may be able to draw more insights from an advanced model.

\section{References}

[1] Amsel, A. 1967. Partial reinforcement effects on vigor and persistence: Advances in frustration theory derived from a variety of within-subjects experiments. In K. W. Spence \& J. T. Spence (Eds.), The psychology of learning and motivation: Advances in research and theory (Vol. 1, pp. 1-65). New York: Academic Press.

[2] Chaiken, S. 1980. "Heuristic versus Systematic Information Processing and the Use of Source versus Message Cues in Persuasion," Journal of Personality and Social Psychology (39:5), pp. 752-766.

[3] Chaiken, S., 1987. The heuristic model of persuasion. In M.P. Zanna, J.M. Olson and C.P. Herman eds.,, Social influence: the ontario symposium (Vol. 5, pp. 3-39).

[4] Chaiken, S. and Maheswaran, D., 1994. Heuristic processing can bias systematic processing: Effects of source credibility, argument ambiguity, and task importance on attitude judgment. Journal of personality and social psychology, 66, pp.460-460.

[5] Chen, P., Dhanasobhon, S., and Smith, M.D. 2006. "All Reviews are Not Created Equal", Working Paper.

[6] Chen, Z. and Lurie, N.H., 2013. Temporal contiguity and negativity bias in the impact of online word of mouth. Journal of Marketing Research, 50(4), pp.463-476.

[7] Chevalier, J. and Mayzlin, D. 2006. "The Effect of Word of Mouth on Sales: Online Book Reviews," Journal of Marketing Research (4:3), pp. 345-354.

[8] Chintagunta, P.K. and Haldar, S. 1998. "Investigating Purchase Timing Behavior in Two Related Product Categories", Journal of Marketing Research (35:1), pp. 43-53. [9] Dahlberg, L., 2005. The corporate colonization of online attention and the marginalization of critical communication?. Journal of Communication Inquiry, 29(2), pp.160-180.

[10] Davenport, T.H., and Beck, J.C. 2001. The Attention Economy: Understanding the New Currency of Business, Boston: Harvard Business School Press.

[11] Deci, E.L., 1972. Intrinsic motivation, extrinsic reinforcement, and inequity. Journal of personality and social psychology, 22(1), p.113.

[12] Deci, E. L., \& Ryan, R. M. 1985. Intrinsic motivation and self-determination in human behavior. New York: Plenum.

[13] Dellarocas, C., Zhang, X.M. and Awad, N.F., 2007. Exploring the value of online product reviews in forecasting sales: The case of motion pictures. Journal of Interactive marketing, 21(4), pp.23-45.

[14] Dewan, S. and Ramaprasad, J. 2009. "Chicken and Egg? Interplay Between Music Blog Buzz and Album Sales”, In Proceedings of Pacific Asia Conference on Information Systems.

[15] Dewan, S. and Ramaprasad, J. 2007. "Consumer Blogging and Music Sampling: Long Tail Effects", In Proceedings of INFORMS Conference on Information Systems and Technology (CIST).
[16] Dillon, W.R. and Gupta, S. 1996. "A Segment-level Model of Category Volume and Brand Choice", Marketing Science (15:1), pp. 38-59.

[17] Duan, W., Gu, B. and Whinston, A.B. 2008. "Do Online Reviews Matter? - An Empirical Investigation of Panel Data," Decision Support Systems (45:4), pp. 1007-1016.

[18] Fleming, J.H., Darley, J.M., Hilton, J.L. and Kojetin, B.A., 1990. Multiple audience problem: A strategic communication perspective on social perception. Journal of personality and social psychology, 58(4), p.593.

[19] Forman, C., Ghose, A., and Wiesenfeld, B. 2008. "Examining the Relationship Between Reviews and Sales: The Role of Reviewer Identity Disclosure in Electronic Markets", Information Systems Research (19:3), pp. 291-313. [20] Gao, B., Hu, N. and Bose, I., 2017. "Follow the herd or be myself? An analysis of consistency in behavior of reviewers and helpfulness of their reviews". Decision Support Systems, 95, pp.1-11.

[21] Ghose, A., and Han, S.P. 2009. "A Dynamic Structural Model of User Learning in Mobile Media Content", Working Paper.

[22] Ghose, A. and Ipeirotis, P.G., 2006, December. Designing ranking systems for consumer reviews: The impact of review subjectivity on product sales and review quality. In Proceedings of the 16th annual workshop on information technology and systems pp. 303-310.

[23] Goldhaber, M.H., 1997. The attention economy and the net. First Monday, 2(4).

[24] Gupta, S. 1988. "Impact of Sales Promotions on When, What, and How Much to Buy", Journal of Marketing Research (25:4), pp. 342-355.

[25] Hausman, J. 1978. "Specification Tests in Econometrics", Econometrica (46:6), pp. 1251-1271.

[26] Hausman, J., Hall, B.H., and Griliches, Z. 1984. "Econometric Models for Count Data with an Application to the Patents-R\&D Relationship", Econometrica (52:4), pp. 909-937.

[27] Hennig-Thurau, T., Gwinner, K.P. and Walsh, G. 2004. "Electronic Word-of-Mouth Via Consumer-Opinion Platforms: What Motivates Consumers to Articulate Themselves on the Internet?", Journal of Interactive Marketing (18:1), pp. 38-52.

[28] Jeppesen, L.B., and Frederiksen, L. 2006. "Why Do Users Contribute to Firm-Hosted User Communities?", Organization Science (17:1), pp. 45-64.

[29] Kuan, K.K., Hui, K.L., Prasarnphanich, P. and Lai, H.Y., 2015. What makes a review voted? An empirical investigation of review voting in online review systems. Journal of the Association for Information Systems, 16(1), p.48.

[30] Lerner, J., and Tirole, J. 2002. "Some Simple Economics of Open Source", The Journal of Industrial Economics (50:2), pp. 197-234.

[31] Li, X. and Hitt, L. 2008. "Self Selection and Information Role of Online Product Reviews", Information Systems Research (19:4), pp. 456-474.

[32] Liu, B. and Karahanna, E., 2017. "The Dark Side of Reviews: The Swaying Effects of Online Product Reviews on Attribute Preference Construction". Management Information Systems Quarterly (41:2), pp.427-448. 
[33] Liu, Y. 2006. "Word of Mouth for Movies: Its Dynamics and Impact on Box Office Revenue", Journal of Marketing (70:3), pp. 74-89.

[34] Moe, W.W., and Trusov, M. 2011. "The Value of Social Dynamics in Online Product Ratings Forums", Journal of Marketing Research (48:3), pp. 444-456.

[35] Mudambi, S.M. and Schuff, D., 2010. What makes a helpful review? A study of customer reviews on Amazon.com. Working paper.

[36] Pavlou, P.A. and Dimoka, A., 2006. The nature and role of feedback text comments in online marketplaces: Implications for trust building, price premiums, and seller differentiation. Information Systems Research, 17(4), pp.392414.

[37] Pavlou, P., and Gefen, D. 2004. "Building Effective Online Marketplaces with Institution-based Trust", Information Systems Research (15:1), pp. 37-59.

[38] Raven, Bertram H. 1959. "Social influence on opinions and the communication of related content", The Journal of Abnormal and Social Psychology (58:1), pp. 119-128.

[39] Resnick, P., Zeckhauser, R., Friedman, E., and Kuwabara, K. 2000. "Reputation Systems", Communications of the ACM (43:12), pp. 45-48.

[40] Schlosser, A.E., 2005. "Posting versus lurking: Communicating in a multiple audience context", Journal of Consumer Research, 32(2), pp.260-265.

[41] Shanab, M.E., Peterson, D., Dargahi, S. and Deroian, P., 1981. The effects of positive and negative verbal feedback on the intrinsic motivation of male and female subjects. The Journal of Social Psychology, 115(2), pp.195-205.

[42] Shen, W., Hu, Y. J., and Ulmer, J. R. 2015. "Competing for Attention: An Empirical Study of Online Reviewers' Strategic Behavior," MIS Quarterly (39:3), pp. 683-696.

[43] Sun, M., 2012. "How does the variance of product ratings matter?". Management Science, 58(4), pp.696-707.

[44] Zhang, X. and Dellarocas, C. 2006. "The Lord of the Ratings: How a Movie's Fate is Influenced by Reviews?", in Proceedings of the 27th International Conference on Information Systems (ICIS 2006), Milwaukee, WI, pp. 19591978.

[45] Zhang, X., Dellarocas, C. and Awad N.F. 2004. "Estimating Word-of-Mouth for Movies: The Impact of Online Movie Reviews on Box Office Performance," Paper presented at the 2004 Workshop on Information Systems and Economics (WISE 2004), College Park, MD.

[46] Zhang, X. 2006. "Tapping into the pulse of the market: Essays on marketing implications of information flows," Ph.D. dissertation, MIT, Cambridge, MA. 